\title{
Extending Fermi LAT discoveries: Compton-Pair Production Space Telescope (ComPair) for MeV Gamma-ray Astronomy
}

\author{
Alexander Moiseev*, Elizabeth Ferrara, Roopesh Ojha, Andrew Smith \\ all CRESST/NASA/GSFC and University of Maryland, Greenbelt, Maryland 20771, USA \\ E-mail: alexander.a.moiseev@nasa.gov
}

Elizabeth Hays, John W. Mitchell, Julie McEnery, Jeremy Perkins, Judith Racusin, David Thompson

all NASA/GSFC, Greenbelt, Maryland 20771, USA

\section{James Buckley}

Department of Physics, Washington University, St. Louis, MO 63130, USA

\section{Regina Caputo}

UCSC and SCIPP, Santa Cruz, CA 95064, USA

\section{Marco Ajello, Dieter H. Hartmann}

Department of Physics \& Astronomy, Clemson University, SC 29634, USA

\begin{abstract}
The gamma-ray energy range from a few hundred $\mathrm{keV}$ to a few hundred $\mathrm{MeV}$ has remained largely unexplored, mainly due to the challenging nature of the measurements, since the pioneering, but limited, observations by COMPTEL on the Compton Gamma-Ray Observatory (19912000). This energy range is a transition region between thermal and nonthermal processes, and accurate measurements are critical for answering a broad range of astrophysical questions. We are developing a MIDEX-scale wide-aperture discovery mission, ComPair (Compton-Pair Production Space Telescope), to investigate the energy range from $200 \mathrm{keV}$ to $>500 \mathrm{MeV}$ with high energy and angular resolution and with sensitivity approaching a factor of 100 better than COMPTEL. This instrument will be equally capable to detect both Compton-scattering events at lower energy and pair-production events at higher energy. ComPair will build on the heritage of successful space missions including Fermi LAT, AGILE, AMS and PAMELA, and will utilize well-developed space-qualified detector technologies including Si-strip and CdZnTe-strip detectors, heavy inorganic scintillators, and plastic scintillators.
\end{abstract}

The 34th International Cosmic Ray Conference,

30 July- 6 August, 2015

The Hague, The Netherlands

${ }^{*}$ Speaker. 


\section{Introduction}

Between soft X-rays (less than $10 \mathrm{keV}$ ) and hard gamma rays (greater than $100 \mathrm{MeV}$ ) lies an energy range of transition in the Universe. Thermal sources dominate at lower energies, while non-thermal processes prevail at higher energies. This energy domain remains the most unexplored in gamma-ray astronomy. Missions like NuSTAR and INTEGRAL at the low end of this transition region and Fermi Gamma-ray Space Telescope at the upper end point to the region, particularly between a few $\mathrm{MeV}$ and $100 \mathrm{MeV}$, as being critical for answering a broad range of astrophysical questions [1]. New technologies and the scientific incentives provided by ongoing missions at higher and lower energies have now paved the way for a greatly improved instrument in this energy range, one we call ComPair because it uses both Compton scattering and pair production as detection techniques. Measurement at these energies is challenging. This is mainly due to specifics of photon detection: it is a range where two processes of photon interaction, Compton scattering and pair production, compete, with a crossover at around $10 \mathrm{MeV}$ depending on the material. These two interaction processes require different approaches in both detection and data analysis, and consequently in the instrument concept. The ideal would be to have two separate instruments, each optimized for a particular photon interaction. However, we believe that it is possible, though challenging, to design a cost-saving single instrument that will be capable to detect both kinds of photon interaction processes and provide accurate results in the extended energy range $0.2-500$ $\mathrm{MeV}$, optimizing its performance in the $0.5-100 \mathrm{MeV}$ span. An additional advantage of such an approach is that the Compton and pair-production events will be treated separately, and the results will be compared in the overlapping energy range. This will significantly increase the confidence in the results obtained, and improve the assessment of systematic errors.

\section{ComPair Instrument Concept}

The instrument concept is based on the traditional scheme of previous gamma-ray telescopes (e.g. EGRET [2], Fermi-LAT [3], AGILE [4]), as well as on the engineering studies for the European-led MEGA [5] and GRIPS [6] projects and utilizes well-known detectors without any consumables. It consists of a multi-layer silicon-strip Tracker in which the gamma-rays interact and the resulting charged particles have directions and energy measured; a Calorimeter to measure deposited energy and position of photon absorption, consisting of a novel combination of solidstate detectors CdZnTe-strip (CZT), and inorganic scintillators CsI(Tl); and a surrounding plastic scintillator anticoincidence detector (ACD), to discriminate the photons from the vastly more numerous charged particles in the space environment (Fig.1).

For pair-production events, ComPair is similar in design to AGILE and Fermi-LAT, but optimized for lower energy. This goal can be achieved by eliminating the passive tungsten converters used in both these instruments. This approach reduces gamma-ray conversion efficiency (roughly proportional to the tracker layer thickness), but it improves the instrument point-spread function (PSF) by reducing absorption and multiple Coulomb scattering of the electron and positron. The broad PSF is a primary limiting factor in the science that can be done at energies below $100 \mathrm{MeV}$ by AGILE and Fermi-LAT. In addition to improving the PSF, the use of low-mass tracker planes also enables photon polarization measurements. 


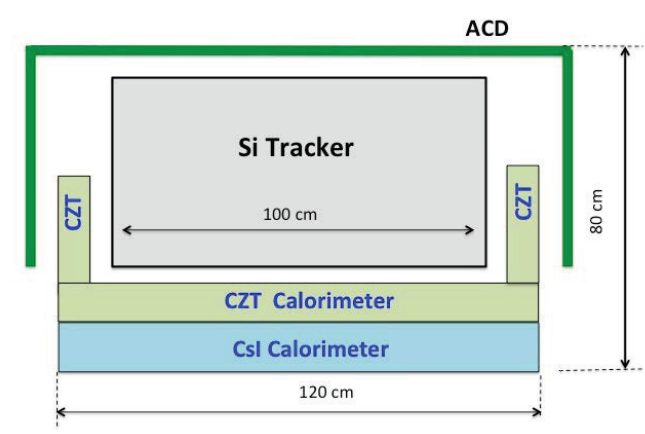

Figure 1: Conceptual design of ComPair

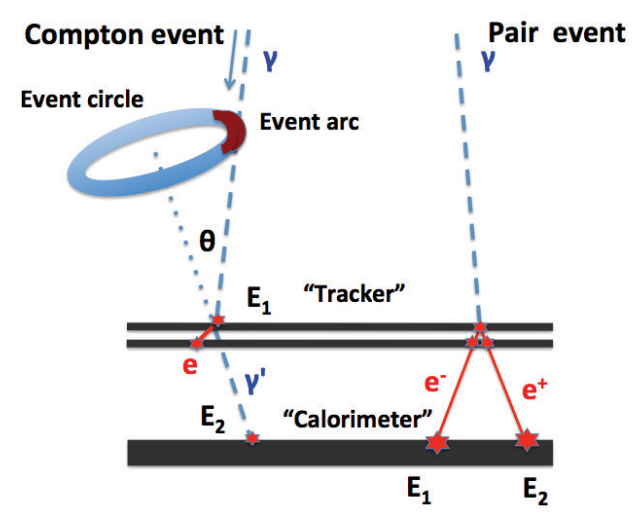

Figure 2: Event types for Compton-Pair telescopes.

Detecting gamma-rays by Compton scattering (the dominant process below about $10 \mathrm{MeV}$ ) is more complicated than using pair production, because the scattered photon carries a significant amount of the information about the incident photon and so needs to be detected too. In practice, a Compton telescope requires two separate photon interactions in order to have a clear detection. In the ComPair concept, the first Compton scattering of the incident photon occurs in one of the Tracker planes, creating an electron and a scattered photon. The Tracker measures the interaction location, the electron energy, and in some cases the electron direction. The scattered photon can be absorbed in the Calorimeter or scattered a second time in the Tracker before being absorbed in the Calorimeter where its energy and absorption position are measured.

The basic principle of the Compton mode of operation is illustrated in Figure 2, left. An incident gamma-ray Compton scatters by an angle $\Theta$ in one layer of the Tracker, transferring energy $E_{1}$ to an electron. The scattered photon is then absorbed in the Calorimeter, depositing energy $E_{2}$, and the scattering angle is given by $\cos \Theta=\frac{m_{e} c^{2}}{E_{2}}+\frac{m_{e} c^{2}}{E_{1}+E_{2}}$. With this information, we derive an "event circle" from which the original photon arrived. We will call this sort of Compton events "untracked" events. The uncertainty in the "event circle" reconstruction is reflected in its width and is due to the uncertainties in direction reconstruction of the scattered photon and the energy measurements of the scattered electron $\left(E_{1}\right)$ and the scattered photon $\left(E_{2}\right)$. If the scattered electron direction is measured, the event circle reduces to an event arc with length due to the uncertainty in the electron direction reconstruction, allowing improved source localization. This event is called a "tracked" event, and its direction reconstruction is somewhat similar to that for pair event: the primary photon direction is reconstructed from the direction and energy of two secondary particles: scattered electron and photon.

\subsection{Si-strip Tracker}

Silicon strips have become the standard particle tracking technology on the ground and in space. Double-sided silicon detectors (DSSD) are necessary for measuring both $\mathrm{x}$ - and $\mathrm{y}$-coordinates of Compton event starting points (low-energy Compton electrons may not penetrate two singlesided detectors) and for measuring polarization for pair-production events: 
- Analog readout is necessary for measurement of the energy of the scattered Compton electron, as well as for measuring energies of pair-production events at low energy.

- The thickness of Si needs to be optimized between conversion efficiency (affects instrument effective area) and multiple scattering (affects PSF).

- Si-strip pitch needs to be optimized between PSF (for Compton events, and for pair-production events at higher energy) and number of electronics channels (cost, power).

- Si-strip plane spacing needs to be optimized between PSF for pair-production events at higher energy and the instrument field of view.

The Tracker is a stack of 50 double-sided $0.5 \mathrm{~mm}$ thick Si-strip detector planes, with an area of $1 \mathrm{~m} \times 1 \mathrm{~m}$ and strip pitch $0.25 \mathrm{~mm}$. The planes are separated by $1 \mathrm{~cm}$, which we currently consider optimal in terms of the PSF/field-of-view trade-off. Each plane is made of $10 \times 10$ DSSD wafers, $9.5 \mathrm{~cm} \times 9.5 \mathrm{~cm}$ each, divided in four $5 \times 5$ wafer segments with daisy-chained strips in each direction (within one segment). In this layout the readout is arranged on the Tracker sides (tentatively by IdEAS VATA4560.3), without the need to have mechanically separated "towers" (Fig. 3).

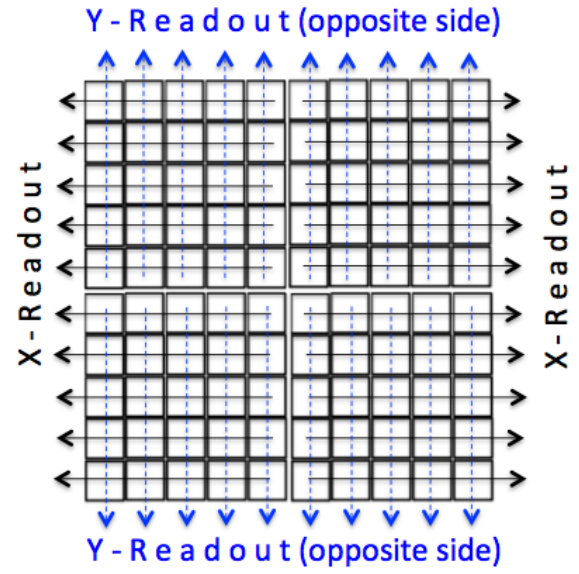

Figure 3: Si Tracker single plane, divided in 4 segments, with $5 \times 5$ wafers in each ( $25 \times 25$ for CZT plane)

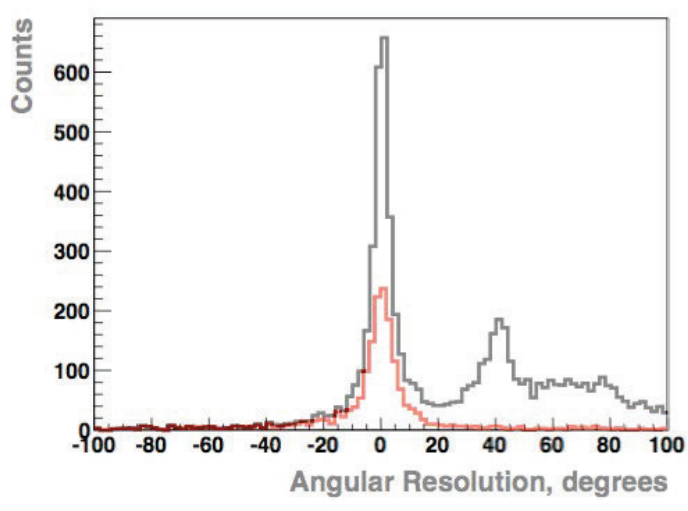

Figure 4: Direction reconstruction for normal incidence $800 \mathrm{keV}$ photons. Red line shows distribution for tracked events, and black line - both tracked and untracked events (see explanation in the text).

\subsection{Calorimeter}

In order to provide necessary information for both Compton and pair-production events over a broad energy range, the Calorimeter needs to have both good spatial and energy resolution over a broad energy range. For the parts closest to the Tracker, where knowing the positions of the interacting low-energy Compton-scattered photons is important, the Calorimeter should be position sensitive with $\sim 5 \mathrm{~mm}$ position resolution in all 3 dimensions and have energy detection threshold less than $50 \mathrm{keV}$, In order to measure the higher-energy pair-production particles, a deeper 
Calorimeter is needed. Potentially it can be made less expensive with coarser position resolution $(1-2 \mathrm{~cm})$ with a higher energy detection threshold of $>100 \mathrm{keV}$. However, the thicker a precise coordinate-sensitive section of the calorimeter is (but more expensive), the better the instrument performance for Compton events (see Section 3 and Fig. 5, right panel).

The ComPair calorimeter is divided into 3 sections (Fig. 1). CZT-strip calorimeters at the bottom and sides detect low-energy Compton-scattered photons, while below the bottom-most CZT detector is a Fermi LAT-like CsI hodoscopic log calorimeter to provide energy measurement and reconstruction of longitudinal and latitudinal shower profiles for higher-energy pair-production events. The total thickness of the bottom section of the calorimeter is $\sim 4.5$ radiation lengths. The CZT calorimeters are made of 4 planes of $5 \mathrm{~mm}$ thick double-sided CZT-strip detectors. Each plane is made of $2 \mathrm{~cm} \times 2 \mathrm{~cm} \times 0.5 \mathrm{~cm}$ individual CZT detectors, with four orthogonal readout strips on both sides. The strip pitch is $5 \mathrm{~mm}$, and strip width is $2.5 \mathrm{~mm}$. In the bottom section each plane is divided in 4 segments, similarly to the Tracker plane (Fig. 3). Each segment contains 25 x 25 individual daisy-chained CZT detectors, read out from two adjacent sides (tentatively by IdEAS VATA).

The CsI calorimeter consists of 5 planes (or trays) of $1.2 \mathrm{~cm} \times 1.2 \mathrm{~cm} \times 32 \mathrm{~cm} \mathrm{CsI(Tl)} \operatorname{logs}$, with each log read out from both ends by Silicon Photomultiplier (tentatively Hamamatsu S12572010P). In the top-most plane (tray) the logs are arranged along the $\mathrm{X}$-axis, in the next one - along the Y-axis, and so on alternatively. The physical location of each $\mathrm{CsI}(\mathrm{Tl}) \log$ provides two spatial coordinates for the "hit", or center of gravity of energy deposition in this log. The one is the number of the plane, or Z-coordinate, and the other is the Y-position of the log in the X-tray, or alternatively X-position for the the Y-tray. The third coordinate along the log length is obtained from the asymmetry in light collection from each end of the log, with expected accuracy of $\sim 5$ $\mathrm{mm}$. An important feature of the ComPair calorimeter is the ability to reconstruct the shower profile using measured deposited energy in each log and its coordinates, similarly to how it is done in Fermi-LAT [3]. This improves energy measurement at higher energies by providing the ability to correct for energy leaking through the bottom and sides of the calorimeter. The shower shape is also an important discriminant between the electromagnetic showers produced by gamma-rays and hadronic showers produced by cosmic-ray background.

\subsection{Anti-Coincidence Detector and Trigger}

The entire upper part of the instrument is covered by a plastic scintillator ACD, similar to that successfully used in Fermi-LAT. The main distinctive feature of the Fermi-LAT ACD is that it is divided in 89 segments in order to reduce a "back-splash" effect at high energy (above 100 $\mathrm{GeV}$ ). It will not be a problem for ComPair due to the lower energy of interest, so the ACD design is significantly easier. The baseline trigger is a five-fold coincidence of the hits in two consecutive Si planes (both sides in each plane) and the presence of a signal from the calorimeter, either CZT or CsI section, with no veto signal in the ACD within the triggering time window of a few microseconds. This trigger configuration allows detection of both Compton and pair-production events. The requirement of hits in two consecutive Si planes assumes the detection of the scattered electron for Compton events. The trigger can be re-configured to require a hit in only one Si plane and in the calorimeter (three-fold coincidence), subject to the orbital event data rate. This trigger configuration allows for the detection of "untracked" Compton events (see Section 3). 


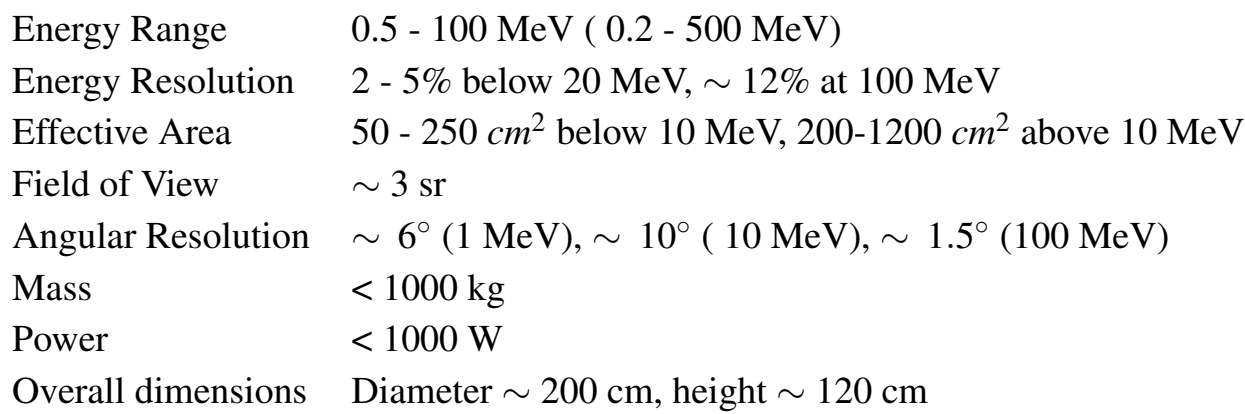

Table 1: Summary of ComPair parameters

\section{Instrument Performance}

Instrument performance was simulated with MEGAlib - the Medium-Energy Gamma-ray Astronomy library [7] and cross-checked with MGEANT [8]. MEGAlib is based on GEANT4 and has been successfully used for numerous simulations, including projects similar to ComPair: MEGA, GRIPS and ASTROGAM. It consists of two main logical parts: one simulates the events, and the other one reconstructs the events and analyzes the results. Event reconstruction for the pair events is rather straightforward, but it is more difficult for the Compton events. As was stated in the instrument trigger description, we will mainly use Compton "tracked" events with a scattered electron detected in at least two Si planes. Using such events we will have smaller effective area and higher detection threshold than for "untracked" events without detected Compton electron, but the direction reconstruction is more reliable (Fig.4). The sample of "untracked" events has many events with misreconstructed directions (seen on the right from the central peak in this figure) which constitutes a significant source of background. Example of such background is atmospheric gamma-radiation arriving mainly from the horizon. However, for some specific observations, e.g. study of spatial structure of a source, the use of untracked events can be useful. Simulation results for effective area and angular resolution for normal incidence events, separately for Compton and pair events, are shown in Fig. 5, reflecting the effect of tracked and untracked events, along with characteristics for Fermi-LAT shown for comparison. We also investigated the effect of the thicker section of CZT calorimeter with better spatial resolution on the ComPair angular resolution. The dotted line (3) on the right panel of Fig. 5 shows angular resolution for the instrument with the CZT calorimeter section consisting of 10 planes, to be compared with the baseline design with 4 planes (solid blue line 1). The effect is visible, but the cost of such a calorimeter would be significantly higher.

Fig. 6 shows the ComPair energy resolution. Clearly seen is the worsening of the resolution at around 3-5 MeV due to the events with a $511 \mathrm{keV}$ annihilation photon escaping from the calorimeter. We have not implemented yet any shower shape reconstruction to improve energy reconstruction, so this is the conservative estimate of ComPair ability to measure the photon energy.

The source sensitivity $I_{s r c}$ for the ComPair instrument compared with existing results is shown in Fig.7 (data for this figure are taken from [6] with added ComPair sensitivity). For Compton events we used only "tracked" events, which direction reconstruction is similar to the pair events. We expect an improvement in sensitivity by a factor of $\sim 100$ compared with that of COMPTEL. It was calculated as 

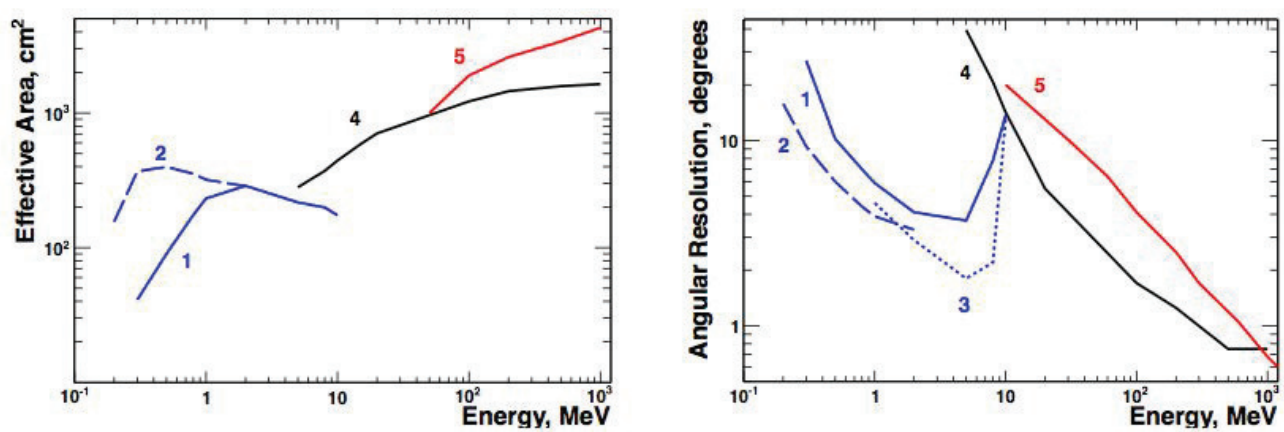

Figure 5: Left panel: Effective area. Right panel: Angular resolution, FWHM for Compton events and $68 \%$ containment radius for pair events. Line 1: Compton tracked events; line 2: Compton untracked events; line 3: Compton tracked events with deeper CZT calorimeter; line 4: pair events with ComPair; line 5: pair events with Fermi-LAT, front.

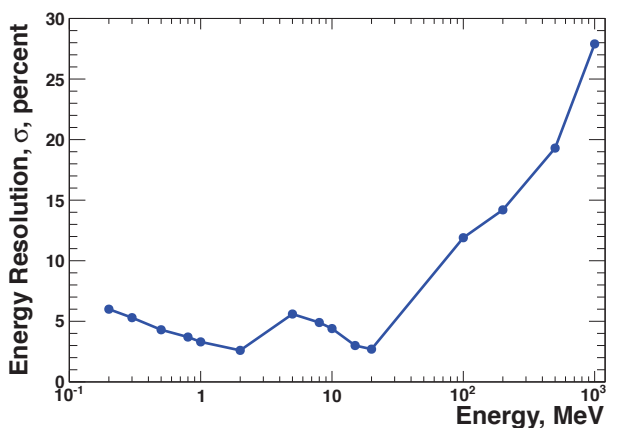

Figure 6: Energy Resolution for ComPair

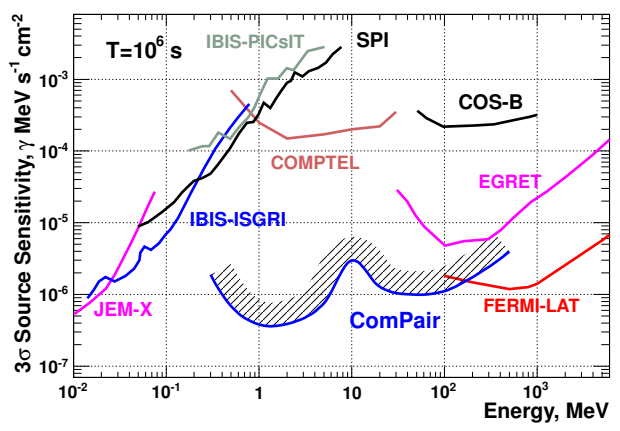

Figure 7: The sensitivity of ComPair mission, compared with other measurements

$$
I_{s r c}=\frac{E}{A \times T} \times\left[\frac{n_{\sigma}^{2}}{4}+\sqrt{\frac{n_{\sigma}^{2} \times B_{b c k g} \times A \times T \times \Delta \Omega}{E}}\right] M e V s^{-1} \mathrm{~cm}^{-2}
$$

where $B_{b c k g}$ was taken as the extragalactic background (EGB) radiation [10], $T=10^{6} \mathrm{~s}$, A is the effective area, and $\Delta \Omega$ is the angular resolution (solid angle). However, the "natural" background such as isotropic EGB for high latitude, and Galactic diffuse radiation for low latitude observations is not the main limiting factor in the instrument sensitivity. Compton events are also affected by the atmospheric and environmental (created in the instrument and spacecraft material) backgrounds. Accurate study of these, and possibly other sources of background, is the subject of a separate work. In this paper we estimated the ComPair source sensitivity, assuming only diffuse EGB background according to the above formula (Fig.7, ComPair blue line). We also estimated the systematic uncertainty, arbitrarily assuming ten times higher background, believed to be the worst case performance with background rejection. It is shown in Fig. 7 as a shadowed area above the ComPair line. This is a preliminary estimate of the ComPair sensitivity, subject to future revision by adding more fidelity to the simulations.

The ComPair capability to detect radiation polarization from bright sources has been estimated 
(for pair events only) for the Crab pulsar based on calculations in [9]. The major factor affecting the polarization measurement for pair events by pair-converter telescopes is the multiple scattering of the electron and positron in the converter, which is a Si-strip plane in ComPair. Although the Si-strip plane thickness of $0.5 \mathrm{~mm}$ is not optimal, it still allows for such measurements. We estimated the ComPair sensitivity to measure linear polarization from the Crab, assuming measured asymmetry parameter $\lambda=0.2$ (corresponding to the converter thickness). If the Crab polarization is $20 \%$, it will be detected by ComPair in 1 month of observation time with $30 \%$ accuracy.

\section{Summary}

We have developed a concept for a medium-energy gamma-ray space telescope, focused on accurate measurements in the energy range $500 \mathrm{keV}-100 \mathrm{MeV}$, with capability to perform measurements in an extended range from $200 \mathrm{keV}$ to $500 \mathrm{MeV}$ and higher. It inherits from predecessors such as Fermi-LAT, AGILE and MEGA. The ComPair concept assumes detecting capability for both Compton and pair production gamma-ray events and exceeds the currently achieved sensitivity of COMPTEL by a factor of $\sim 100$ at energy around $1 \mathrm{MeV}$. The design presented in this paper can be considered as a concept with the possibility to scale it up or down depending on the available mission resources. The authors would like to thank Robert Johnson, Bill Atwood, Eric Grove, Eric Wulf and Steve Sturner for valuable discussions and suggestions. Also the authors are grateful to the MEGA team, and especially to Andreas Zoglauer for the wonderful simulation package MEGAlib.

\section{References}

[1] J. McEnery et al., same Proceedings

[2] Thompson, D. J., et al. 1993, ApJS, 86, 629

[3] Atwood, W. B. et al. (2009, June). The Large Area Telescope on the Fermi Gamma-Ray Space Telescope Mission. ApJ 697, 1071

[4] Tavani, M. et al. (2009, August). The AGILE Mission. AAP 502, 995

[5] Bloser, P. F., R. Andritschke, G. Kanbach, V. Schönfelder, F. Schopper, and A. Zoglauer. The MEGA advanced Compton telescope project. NAR 46, 611, 2002

[6] J. Greiner at al., Gamma-ray Imaging, Polarimetry and Spectroscopy, Experimental Astronomy, October 2012, Volume 34, Issue 2, 551

[7] A. Zoglauer, R. Andritschke, and F. Schopper, MEGAlib - The Medium Energy Gamma-ray Astronomy Library, New Astronomy Reviews 50, 2006, 629

[8] S. J. Sturner, H. Seifert, C. Shrader, and B. J. Teegarden, MGEANT - A GEANT-based multi-purpose simulation package for gamma-ray astronomy missions, AIP Conf. Proc. 510, 814, 2000

[9] S.R. Kelner, Yu.D. Kotov, and V.M. Logunov, Methods of measuring linear polarization of gamma-quanta, Sov. J. Nuclear Physics, 21, 3, 604, 1975

[10] A. Strong, I. Moskalenko, and O. Reimer, ApJ 537, 763, 2000 